\title{
Simplified LS and Modified MMSE Estimators for Channel Estimation of OFDM
}

\author{
B. Janardhana Rao ${ }^{1}$ and O. Venkata Krishna ${ }^{2}$ \\ ${ }^{1}$ CVR College of Engineering/ECE, Hyderabad, India \\ Email: janardhan.bitra@gmail.com \\ ${ }^{2}$ CVR College of Engineering/EIE, Hyderabad, India \\ Email: venkatakrishna.odugu@gmail.com
}

\begin{abstract}
In this paper we investigate the blocktype pilot channel estimation for orthogonal frequency division multiplexing (OFDM) systems. The estimation is based on the minimum mean square error (MMSE) estimator and the least square (LS) estimator. We derive the MMSE and LS estimators' architecture and investigate their performances. We prove that the MMSE estimator performance is better but computational complexity is high, contrary the LS estimator has low complexity but poor performance. For reducing complexity we proposed two different solutions which are the Simplified Least Square (SLS) estimator and the modified MMSE estimator. In the SLS estimator, we apply an auto-correlation function with the LS estimator to remove the noise. In the modified MMSE estimator, we consider only the significant energy samples and ignore the remaining noisy samples. Based on this idea we introduce the modified MMSE estimator. We evaluate estimator's performance on basis of mean square error and symbol error rate for 16 QAM systems using MATLAB.
\end{abstract}

Index Terms-Channel Estimation, OFDM, LS Estimator, MMSE Estimator, SLS estimator

\section{INTRODUCTION}

Orthogonal Frequency Division Multiplexing (OFDM) is one of the most widely used modulation technique for high-bit-rate wireless communication. Especially the wireless local area network systems such as WiMax, WiBro, WiFi and the emerging fourthgeneration mobile systems are used OFDM as the core modulation technique. Wireless communication systems use two different signaling schemes which are: coherent and general signaling schemes. Quadrature Amplitude Modulation (QAM) which is Coherent signaling scheme requires channel estimation and tracking of the fading channel.

In OFDM system, the channel is usually assumed to have a finite impulse response. To avoid the intersymbol interference, a cyclic extension is put between the consecutive blocks, where the cyclic extension length is longer than the channel impulse response.
Decision-directed and pilot-symbol-aided methods are two different ways for channel estimation. Pilotsymbol-aided channel estimation can be further divided in two types: block type-pilot channel estimation and comb-type-pilot channel estimation. All sub-carriers are reserved for the pilot within a specific period in blocktype-pilot method. The estimation of the channel can be based on Least Square (LS) or on Minimum Mean Square Error (MMSE)

in this method. In the comb-type-pilot method, one subcarrier is reserved as a pilot for each symbol. The estimation of the channel for the comb-type-pilot arrangement can be based on linear interpolation, second order interpolation, low-pass interpolation or on time domain interpolation.

The MMSE estimator performance is good but its complexity is high. Contrary the LS estimator complexity is low but its performance is poor [1]. For reducing complexity of the both estimators we proposed two different algorithms which reduce complexity without compromise in performance or with slightly lower performance.

\section{OFDM SYSTEM DESCRIPTION}

The basic idea underlying OFDM systems is the division of the available frequency spectrum into several subcarriers, converting a frequency-selective channel into a parallel collection of frequency at sub channels [2]. To obtain a high spectral efficiency, the signal spectra corresponding to the different subcarriers overlap in frequency, and yet they have the minimum frequency separation to maintain orthogonality of their corresponding time domain waveforms [3]. To preserve the orthogonality of the tones and eliminates ISI between consecutive OFDM symbols here we use Cyclic Prefix (CP). A block diagram of a baseband OFDM system is shown in Figure 1.

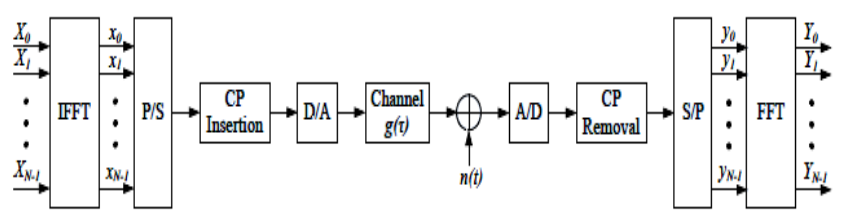

Figure 1: Baseband OFDM 
After the information bits are grouped, coded and modulated, they are fed into N-point inverse fast Fourier transform (IFFT) to obtain the time domain OFDM symbols, i.e.,

$x_{n}=\operatorname{IFFT}_{N}\left\{X_{K}\right\}=\sum_{K=0}^{N-1} X_{K} e^{j 2 \pi K n / N}, 0 \leq n, K \leq N-1$

, where $\mathrm{n}$ is the time domain sampling index, $X_{K}$ is the data at kth subcarrier, and $\mathrm{N}$ is the total number of subcarriers. Following IFFT block, a cyclic extension of time length, $\mathrm{T}_{\mathrm{G}}$, chosen to be larger than the expected maximum delay spread of the channel [5], is inserted to avoid intersymbol and intercarrier interferences. The digital-to-analog (D/A) converter contains low-pass filters with bandwidth $1 / T_{S}$, where $T_{S}$ is the sampling interval or an OFDM symbol period. The channel is modeled as an impulse response, $\mathrm{g}(\tau)$, followed by the complex additive white Gaussian noise $(\mathrm{AWGN}), \mathrm{n}(\mathrm{t})[6]$.

$$
g(\tau)=\sum_{m=0}^{M-1} \alpha_{m} \delta\left(\tau-\tau_{m} T_{s}\right)
$$

, where $\mathrm{M}$ is the number of multipaths, $\alpha_{m}$ is the $m$ th path gain in complex, and $\tau_{m}$ is the corresponding path delay. The N-point FFT is used to transform the data back to frequency domain At the receiver, after passing through the analog-to-digital (A/D) and removing $\mathrm{CP}$. Finally, the information bits are obtained after the channel equalization/decoding, and demodulation.

Under the assumption that the use of a CP preserves the orthogonality of the tones and the entire impulse response lies inside the guard interval, i.e., $0 \leq \tau_{\mathrm{m}} \mathrm{Ts} \leq$ $\mathrm{T}_{\mathrm{G}}[7,9]$, we can describe the received signals as

$$
Y=F_{N}\left\{T_{N F T}\{X\} \otimes g+\tilde{n}\right\}
$$

, where $Y=\left[Y_{0} Y_{1} \ldots \ldots Y_{N-1}\right]^{T}$ is the received vector, $X$ $=\left[\begin{array}{lll}X_{0} & X_{1} \ldots \ldots . X_{N-1}\end{array}\right]^{T}$ is a vector of the transmitted signal, and $\mathrm{g}=\left[\begin{array}{llll}\mathrm{g}_{0} & \mathrm{~g}_{1} & \ldots . . \mathrm{g}_{\mathrm{N}-1}\end{array}\right]^{\mathrm{T}} \quad$ and $\tilde{\mathrm{n}}=\left[\begin{array}{llll}\tilde{\mathrm{n}}_{0} & \tilde{\mathrm{n}}_{1} & \ldots . & \tilde{\mathrm{n}}_{\mathrm{N}-1}\end{array}\right]^{\mathrm{T}}$ are the sampled frequency response of $g(\tau)$ and $A W G N$, respectively. Note that both $\mathrm{Y}$ and $\mathrm{X}$ are frequency domain data. In fact, the expression in equation (3) is equivalent to a transmission of data over a set of parallel Gaussian channels [8], as shown in Figure 2.

Therefore, the system described by equation (3) can be written as

$$
Y=X F_{g}+F \tilde{n}
$$

, where $\mathrm{X}$ is a diagonal matrix containing the elements of $\mathrm{X}$ in equation (3), and

$$
\boldsymbol{F}=\left[\begin{array}{ccc}
W_{N}^{00} & \cdots & W_{N}^{0(N-1)} \\
\vdots & \ddots & \vdots \\
W_{N}^{(N-1) 0} & \cdots & W_{N}^{(N-1)(N-1)}
\end{array}\right]
$$

is the FFT matrix with

$$
W_{N}^{n k}=\frac{1}{\sqrt{N}} e^{-j 2 \pi \frac{n k}{N}}
$$

Also,

$$
\text { let } \quad \mathrm{h}=\mathrm{FFT}_{\mathrm{N}}\{\mathrm{g}\}=\mathrm{Fg} \text { and }
$$

$n=F F T_{N}\{\tilde{n}\}=F \tilde{n}$. Thus, equation (4) now becomes

$$
Y=X h+n
$$

we assume that the noise $\mathrm{n}$ is a vector of independent identically distributed complex zero-mean Gaussian noise with variance $\sigma_{n}^{2}$. We also assume that $\mathrm{n}$ is uncorrelated with the channel $h$.
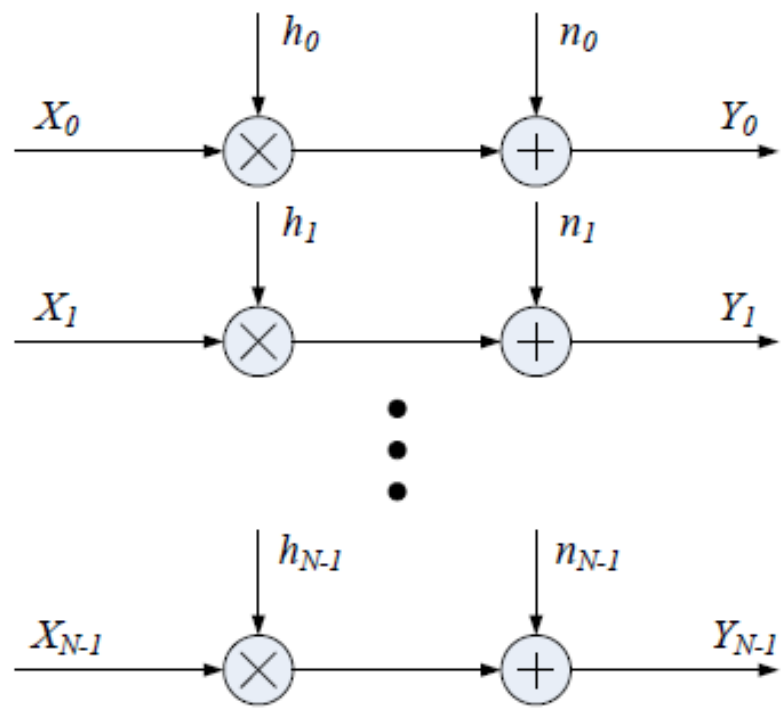

Figure 2: The OFDM system, modeled as parallel Gaussian channels

\section{CHANNEL ESTIMATION TECHNIQUES}

\section{A. MMSE Estimator}

The major rule of MMSE estimator is to efficiently estimate the channel to minimize the MSE or SER of the channel. In equation (5), $R_{g g}$ and $R_{y y}$ denote as the auto-covariance matrix of $\boldsymbol{g}$ and $\boldsymbol{y}$ respectively, where $\boldsymbol{g}$ is the channel energy and $\boldsymbol{y}$ is the received signal. Moreover, the cross covariance of $\boldsymbol{g}$ and $\boldsymbol{y}$ is denoted by $R_{g y}$ and the noise variance $E\left\{|n|^{2}\right\}$ is denoted by $\delta_{n}^{2}$. The channel estimation by using MMSE estimator $g_{M M S E}$ can be derived as follows: 


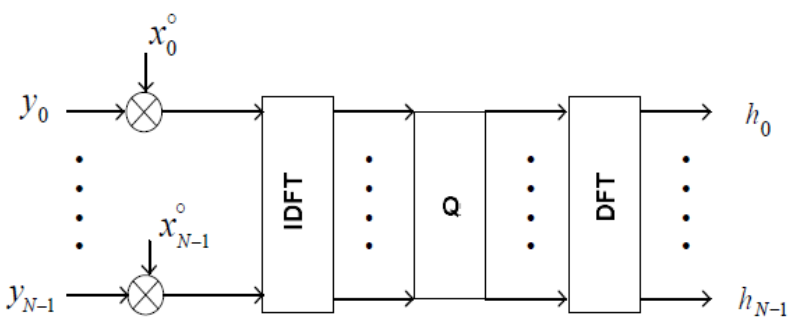

Figure 3: Block diagram of channel estimator

$$
g_{M M S E}=R_{g y} R_{y y}^{-1} y
$$

Where,

$$
\begin{array}{r}
R_{g y}=E\left\{g y^{H}\right\}=R_{g g} F^{H} x^{H} \\
R_{y y}=E\left\{y y^{H}\right\}=x F R_{g g} F^{H} x^{H}+\delta_{n}^{2} I_{n}
\end{array}
$$

The columns in $\boldsymbol{F}$ are orthogonal and $\boldsymbol{I}$ is the identity matrix. From Figure 3, the channel impulse response $h_{M M S E}$ is as fallows

$h_{M M S E}=F g_{M M S E}=F Q_{M M S E} F^{H} x^{H} y$

Where,

$Q_{M M S E}=R_{g g}\left[\left(F^{H} x^{H} x F\right) \delta_{n}^{2}+R\right]^{-1}\left(F^{H} x^{H} F x\right)^{-1}-$

$h_{M M S E}$ is the channel attenuation for MMSE estimator,

$g_{M M S E}$ is the channel energy, $\boldsymbol{y}$ is received signal, $\boldsymbol{x}$ is the transmitted signal and $\boldsymbol{F}$ is the DFT matrix [2].

\section{B. LS Estimator}

The LS estimator has lower computational complexity than MMSE. The LS estimator for the cyclic impulse response $g$ minimizes $(y x F g)(y-x F g)^{H}$ and generates the channel attenuation as bellow

$$
h_{L S}=F Q_{L S} F^{H} x^{H} y
$$

Here,

$$
Q_{L S}=\left(F^{H} x^{H} F x\right)^{-1}
$$

and $(y-x F g)^{H}$ are the conjugate transpose operations. So, the lest square $h_{L S}$ can be written as

$$
h_{L S}=x^{-1} y
$$

Where, the least square $h_{L S}$ is the channel attenuation for LS. Equations (9) and (13) are the general expressions for MMSE and LS estimators respectively. The performances of the estimators are evaluated using Mean square error and symbol error rate.

\section{Mean Square Error (MSE)}

The mean square error or MSE of an estimator is one of many ways to quantify the difference between the theoretical values of an estimator and the true value of the quantity being estimated. MSE measures the average of the square of the error. The error is the amount by which the estimator differs from the quantity to be estimated. We define the mean square error as [10]

$$
\text { meansquarerror }=\text { mear }\left\{a b s(H)-a b s\left(h_{\text {estimator }}\right)\right\}^{2}
$$

Where, $\boldsymbol{H}$ is theoretical transfer function and $h_{\text {estimator }}$ is the calculated transfer function for each estimator.

\section{Symbol Error Rate (SER)}

Symbol rate is the number of symbol changes made to the transmission medium per second using a digitally modulated signal. Symbol error rate for 16-QAM system is [11]

$$
P_{S, 16-Q A M}=3 / 2 \operatorname{erfc}\left(\sqrt{\frac{E_{S}}{10 N_{0}}}\right)
$$

Where, erfc denoted complementary error function, $E_{S}$ denoted signal energy and $N_{0}$ denoted bit rate.

Both estimators have some drawbacks. However the MMSE estimator performance is better but computational complexity is high, contrary the LS estimator has high mean-square error means least performance but its computational complexity is very low [2]. For reducing computational complexity and improve performance, we proposed two channel estimation approaches.

\section{MODEL OF THE PROPOSED ESTIMATOR}

\section{A. System Structure for SLS Estimator}

The LS estimator has least performance with high mean square error. For improving the performance and to reduce the computation complexity, we proposed the following SLS estimator.

Equation (12) can be rewrite like this

$$
h_{L S}=h+n
$$

Here

$$
h=F g
$$

$h$ is the transfer function, $n$ is the Gaussian noise, $\boldsymbol{F}$ is the DFT matrix, $g$ is the channel impulse response in time domain. From equation (16), the LS estimator consists of channel transfer function plus some noise. Due to noise part the LS estimator gives the poor performance. The noise from the original signal has to remove to improve the performance. The LS estimation is noisy observation of the channel attenuation which can be smoother using some auto-correlation operation with the channel attenuation $h_{L S}$. If the channel transfer function is $h$, the received signal $y$ and the transmitted symbol $x$, then the SLS channel estimator will be: 


$$
h_{S L S}=W_{x} h_{L S}
$$

where, $W_{x}$ is weighted matrix and

$$
\begin{array}{r}
W_{x}=R_{h h}\left(R_{h h}+\sigma_{n}^{2}\left(x x^{H}\right)^{-1}\right)^{-1} \\
R_{h h}=E\left\{h h^{H}\right\}
\end{array}
$$

where, $R_{h h}$ is the auto-covariance matrix of $h$. The weighting matrix $W_{x}$ of size $N \times N$ depends on the transmitted signal $\boldsymbol{x}$. As a step towards the lowcomplexity estimators we want to find a weighting matrix which does not depend on the transmitted signal $x$. The weighting matrix can be obtained from the auto-covariance matrix of $h$ and auto-correlation of transmitted signal $x$. Consider that the transmitted signal $x$ to be stochastic with independent and uniformly distributed constellation points. In that case the auto-covariance matrix of noise becomes

$$
R_{n n}=\frac{\alpha}{S N R} I
$$

where, $\quad \alpha$ is constellation factor and $E\left\{\left|X_{i}\right|^{2}\right\} E\left\{1 /\left|X_{i}\right|^{2}\right\}$ is the mathematical expression of $\alpha$. The value of $\alpha$ is $\frac{17}{6}$ for 16-QAM. SNR is a persymbol signal-to-noise ratio equal to $E\left\{\left|X_{i}\right|^{2}\right\} / \delta_{n}^{2}$. Then the SLS estimator becomes

$$
h_{S L S}=W_{\text {mod ified }} h_{L S}
$$

where the modified weighting matrix is give by

$$
W_{\text {modified }}=R_{h h}\left(R_{h h}+R_{n n}\right)^{-1}
$$

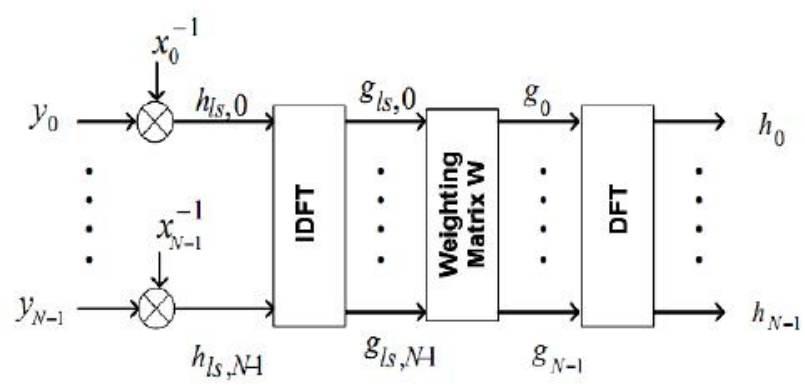

Figure 4: Block diagram for SLS estimator

Figure 4 shows the block diagram of $h_{S L S}$ estimator. $x_{n}$ represents the input signal start from 0 to $N, y_{n}$ is the output from 0 to $N$ sample, $g_{n}$ is the channel impulse response in the time domain from 0 to $N$ samples and $h_{n}$ is the channel transfer function in the frequency domain.

\section{B. System Structure for Modified MMSE}

The modified estimator is based on MMSE estimator. According to equation (2), most of the channel energy $g$ is contained in or near to the first $(L+1)$ samples, where $L$ is $\left[\frac{T_{G}}{T_{S}}\right] N$, where $T_{G}$ is the cyclic extension of time length, $T_{S}$ is sampling interval and $N$ is the DFT size. Therefore to modify the estimator we consider only the significant energy samples that are the upper left corner of auto-covariance matrix $R_{g g}$. From the IEEE std. 802.11 and IEEE std 802.16, $\quad\left[\frac{T_{G}}{T_{S}}\right] \quad$ should be chosen among $\{1 / 32,1 / 16,1 / 8\}$. Considering the significant energy level is 8 . So $\frac{T_{G}}{T_{S}}=1 / 8$ and $L=\frac{1}{8} \times 64=8$. So, the significant energy consists of 1 to 8 sample and remaining samples are noise of low SNR. To reduce the complexity we consider only the significant energy samples.

Figure 5, shows the general structure of Modified MMSE estimator. where $x_{n}$ is the input signal, $y_{n}$ is output signal, $\boldsymbol{Q}$ is frequency response in time domain and $h_{n}$ is the transfer function, all these variables are range from 0 to $N$-th sample.

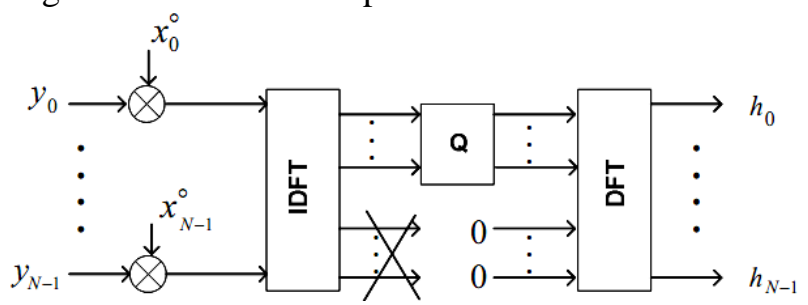

Figure 5: Block diagram for modified MMSE channel estimator

We consider only the significant energy samples that samples are transmit the data signal and remaining samples transmit null signal. In MMSE-3 estimator, first three samples send data signal and remaining samples send null signal. By implement the same approach- , MMSE-5, MMSE-8, MMSE-14 and MMSE-20 estimators' data signal are consists of five, eight, fourteen and twenty samples respectively and the rest of data bit information is set to null signal.

\section{V.SIMULATION AND RESULT ANALYSIS}

The goal of the simulation is efficiently estimate the channel and then validation of the proposed method. The simulation scenarios enable analysis of different channel estimator performance to find the optimal 
channel estimator with low complexity. The significant energy level is one of the major factors to determine estimator performance. In our simulation, the significant energy level is concentrated in the first nine samples. The mean square error and symbol error rate are the major parameters to evaluate the estimators' performance. Our main emphasis is to minimize the mean square error and symbol error rate for each estimator. In our simulation scenario we consider a system with $500 \mathrm{kHz}$ bandwidth which is divided into 64 carriers. The total symbol period is $64 \times 2+10=138$ $\mu \mathrm{s}$ where the symbol period for sender is $64+5=69$ and for receiver $64+5=69$, the system used 64 subcarriers, $10 \mu \mathrm{s}$ is for the cyclic prefix and the sampling is performed with $500 \mathrm{kHz}$ rate. A symbol consists of $64+5=69$ samples where five of them belong to cyclic prefix. Our simulation scenarios are on based the following system parameters are shown in Table 1.

TABLE I

SYSTEM PARAMETERS

\begin{tabular}{|l|l|}
\hline \multicolumn{1}{|c|}{ parameters } & \multicolumn{1}{c|}{ Specification } \\
\hline FFT size & 64 \\
\hline Number of carriers $N$ & 64 \\
\hline Pilot Ratio & $1 / 10$ \\
\hline Guard Length & 10 \\
\hline Guard Type & Cyclic Prefix \\
\hline Bandwidth & $500 \mathrm{KHz}$ \\
\hline Signal Constellation & 16 QAM \\
\hline
\end{tabular}

Numbers of sample in each channel estimator used in our simulation are given in Table 2 .

TABLE II

DIFFERENT CHANNEL ESTIMATORS AND THEIR SIZE

\begin{tabular}{|l|l|l|}
\hline \multicolumn{1}{|c|}{ Estimator } & \multicolumn{1}{|c|}{ Notation } & \multicolumn{1}{c|}{$\begin{array}{c}\text { Number of } \\
\text { sample }\end{array}$} \\
\hline MMSE & MMSE & $0 \ldots \ldots \ldots \ldots \ldots \ldots .63$ \\
\hline LS & LS & $0 \ldots \ldots \ldots \ldots \ldots \ldots 63$ \\
\hline SLS estimator & SLS & $0 \ldots \ldots \ldots \ldots \ldots \ldots 63$ \\
\hline \multirow{2}{*}{$\begin{array}{l}\text { Modified } \\
\text { MMSE }\end{array}$} & MMSE-3 & $0 \ldots .2$ \\
\cline { 2 - 3 } & MMSE-5 & $0 \ldots \ldots .4$ \\
\cline { 2 - 3 } & MMSE-8 & $0 \ldots \ldots \ldots$. \\
\cline { 2 - 3 } & MMSE-14 & $0 \ldots \ldots \ldots \ldots 13$ \\
\cline { 2 - 3 } & MMSE-20 & $0 \ldots \ldots \ldots \ldots \ldots 19$ \\
\hline
\end{tabular}

All the programs are executed in Matlab simulator and the models validations are done on the basis of two parameter analysis are Mean Square Error and Symbol Error Rate.

\section{A. Analysis of simulation result - Mean Square Error approach}

We analyze the different channel estimators' performance based on mean square error criteria according to equation (14).

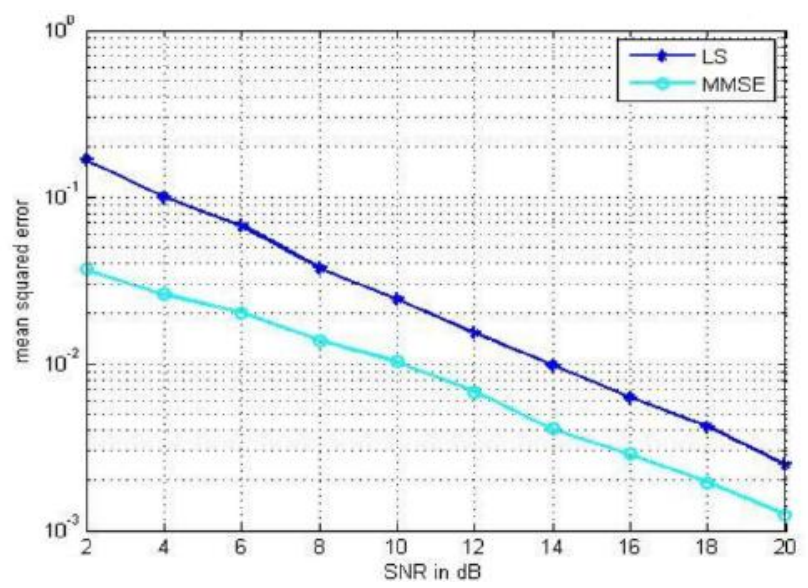

Figure 6: MMSE and LS estimator performance comparison based on characteristics of MSE versus SNR

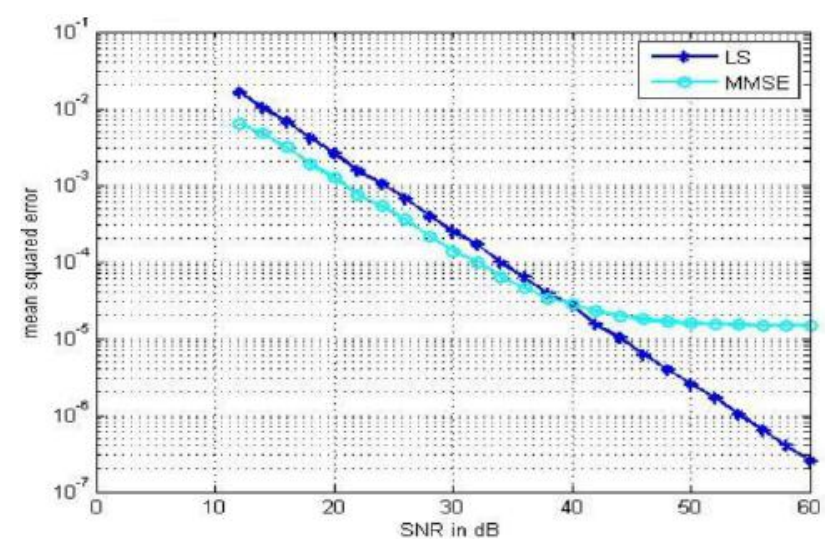

Figure 7: MMSE and LS estimator performance comparison based on characteristics of MSE versus SNR (for higher range of SNR)

Figures 6 and 7 show the mean square error versus SNR curve for LS and MMSE. For SNR range from 2 $\mathrm{dB}$ to $20 \mathrm{~dB}$, the MMSE estimator mean square error range is $10^{-3}$ to $10^{-1}$ whereas the LS estimator mean square error range is $10^{-3}$ to 10 . While SNR range increases from $12 \mathrm{~dB}$ to $60 \mathrm{~dB}$, the MMSE estimator mean square error range is $10^{-5}$ to $10^{-1}$, whereas the LS estimator mean square error range is $10^{-7}$ to $10^{-1}$. LS and MMSE, the both of estimators give lower square error for higher range of SNR. Figures 8 and 9 shows the characteristics of MSE versus SNR for the MMSE, LS and SLS estimators respectively. The SLS estimator performance is better than LS for less than $16 \mathrm{~dB}$ SNR. 


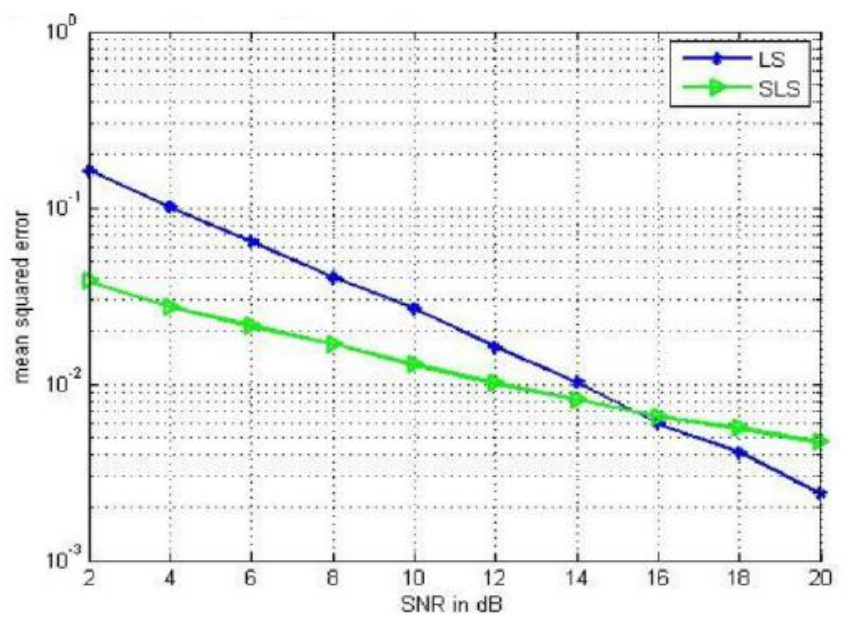

Figure 8: SLS and LS estimator performance comparison based on MSE versus SNR parameters

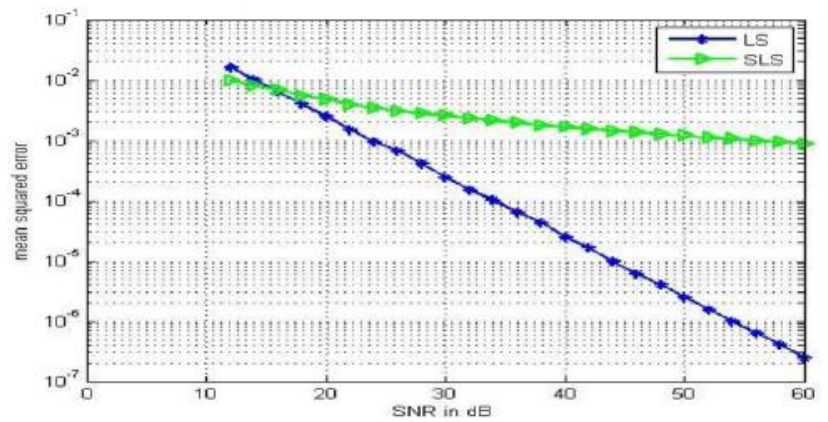

Figure 9: SLS and LS estimator performance comparison based on MSE versus SNR parameters (for higher range of SNR)

Figures 10 and 11 show comparisons of the MSE performance of the estimation schemes with original MMSE and modified MMSE. In Figure 10, the MMSE20 estimator MSE is lower than others modified MMSE and for higher number of power samples estimator gives lower MSE values. In Figure 11, we compare all of modified MMSE estimators with original MMSE estimator where we can observe for higher SNR range, all modified estimators' gives the lower MSE. The original MMSE estimator MSE range is $10^{-5}$ to $10^{-2}$ whereas the modified MMSE estimator MSE range is $10^{-1}$ to $10^{0}$.

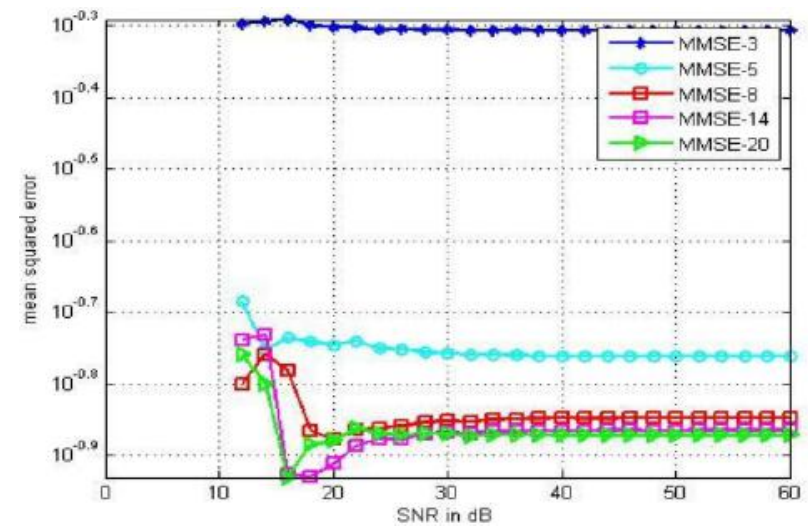

Figure 10: Performance analysis for modified MMSE based on MSE versus SNR

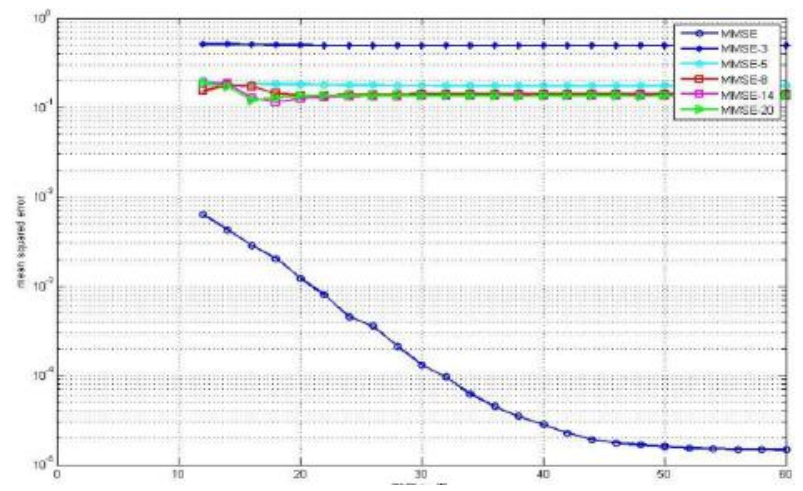

Figure 11: Comparison between Original MMSE and modified MMSE

\section{B. Analysis of simulation result - Symbol Error Rate approach}

In this section, we analysis the different channel estimators' performance based on symbol error rate approach based on equation (15).

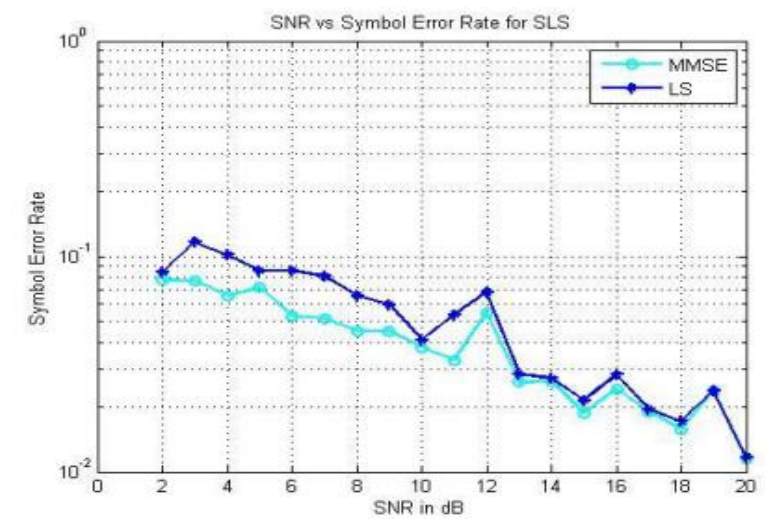

Figure 12: Performance analysis for MMSE and LS based on SER versus SNR

Figures 12 and 13 show the comparison between the LS and MMSE estimator based on SER versus SNR. In the SNR range from $2 \mathrm{~dB}$ to $20 \mathrm{~dB}$, the MMSE estimator SER is lower than the LS estimator. SERs of LS and MMSE are almost the same from $25 \mathrm{~dB}$ SNR range.

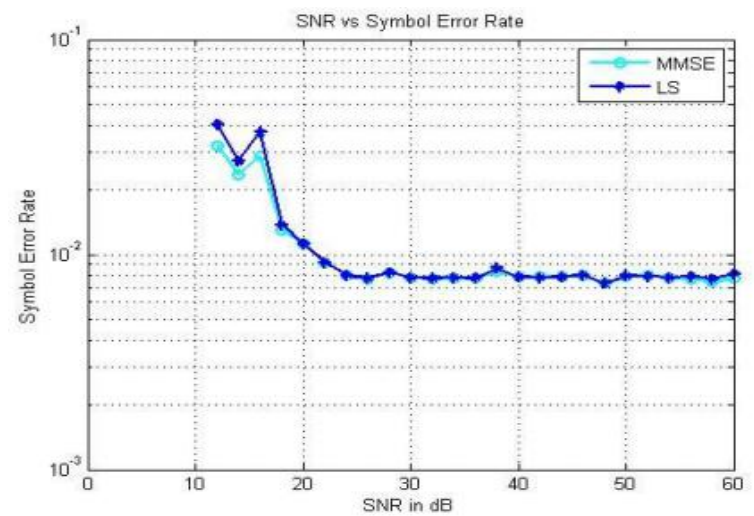

Figure 13: MMSE and LS estimator performance comparison based on characteristics of SERversus SNR (For higher SNR range) 




Figure 14: Performance comparison for SLS and LS based on SER versus SNR

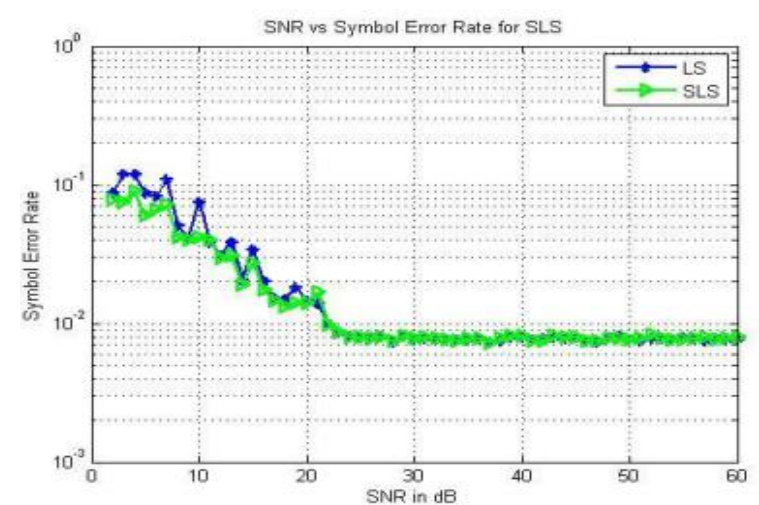

Figure 15: Performance comparison for SLS and LS based on SER versus SNR (for higher SNR range).

Figure 14 and 15 show the performance characteristics of LS and SLS estimator. The SNR range from $2 \mathrm{~dB}$ to $20 \mathrm{~dB}$, the SLS and LS estimator SER are in the range from $10^{-2}$ to $10^{\circ}$. The same for the SNR range from $2 \mathrm{~dB}$ to $60 \mathrm{~dB}$, the SLS and LS estimator SERs are in the same range from $10^{-2}$ to $10^{-1}$. For higher range of SNR the SER is almost same for the LS and SLS estimator.

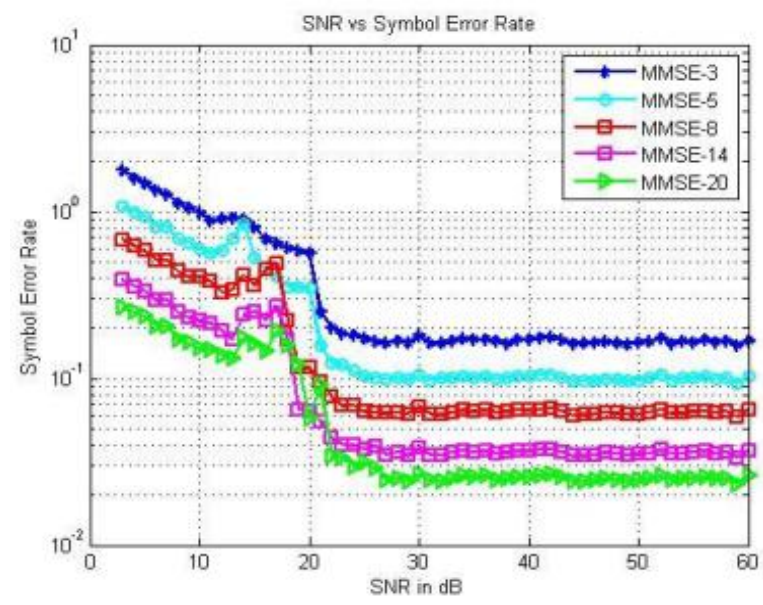

Figure 16: Performance comparison of modified MMSE based on SER versus SNR



Figure 17: Performance comparison of MMSE and modified MMSE based on SER versus SNR

The Figures 16 and 17 illustrate the SER performance of the estimation schemes with original MMSE and modified MMSE. In Figure 16, we can conclude that MMSE-20 estimator SER is lower than all others modified MMSE. When the number of significant energy samples increases, then the SER decreases. So, for larger number of significant energy sample, the performance can be improved. In figure 17, we compare all of modified MMSE estimators' with original MMSE estimator. For higher range of SNR, all of estimator gives lower SER. All of modified MMSE estimators' are in the SER range from $10^{-2}$ to $10^{1}$ whereas the original MMSE estimator SER ranges $10^{-2}$ to $10^{-1}$. It can be concluded that modified MMSE estimator slightly compromises with the performances.

\section{CONCLUSION}

In this paper, first, we show the general structure of all estimators. Then we investigate the LS and the MMSE estimator performances using the mean square error and symbol error rate. Based on the performance analysis the MMSE estimator is recognized as better than LS estimator, but the MMSE estimator suffers from high computational complexity. To reduce its computational complexity we proposed two different channel estimation methods: The SLS estimator and the modified MMSE estimator. The significant energy samples and noisy observation of the LS estimator are the key points to implement our ideas. In the SLS estimator, we apply an auto-correlation function with the LS estimator to remove the noise. In the modified MMSE estimator, we consider only the significant energy samples and ignore the remaining noisy samples. Based on this we introduce the modified MMSE estimator.

By using the Matlab simulator, we validated our models. The comparison of all estimators' performances on basis of mean square error and symbol error rate is shown. The simulation result shows that the MMSE estimator performances better than the LS estimator, especially in higher SNR range. From the performance analysis of each estimator, the SLS estimator MSE is 
$10^{-1}$ to $10^{-7}$ and SER is $10^{-1}$ to $10^{-2}$ for $10 \mathrm{~dB}$ to $60 \mathrm{~dB}$ SNR range. However the modified MMSE estimator MSE is $10^{0}$ to $10^{-1}$ and SER is $10^{1}$ to $10^{-1}$ on the same SNR range. The SLS estimator MSE is lower than the modified MMSE estimator. In modified MMSE estimator, the MMSE-20 estimator gives the lower MSE than the others modified MMSE estimator. In future work, the proposed channel estimation method can be applied for $4 \mathrm{G}$ LTE to achieve high data rate.

\section{REFERENCES}

[1] Yi Gong and K.B Lataief, "Low rank channel estimation for space-time coded wide band OFDM systems", IEEE Vehicular Technology Conference, vol. 2, pp. 772-776, 2001.

[2] Y. Shen and E. Martinez. Channel estimation in ofdm systems. Technical Report AN3059, Freescale Semiconductor, Inc., 2006.

[3] S. W. McLanughln Y. Li M. A. Ingram G. L. Stuber, J. R. Barry and T. G. Pratt. Broadband mimo-ofdm wireless communications. Proceedings of the IEEE, 92(2), Feb. 2004.
[4] Coleri,S; Ergen,M; Puri,A; Bahai,A. "Channel estimation techniques based on pilot arrangement in OFDM systems", IEEE Transactions on Broadcasting, vol. 48, Issue 3, pp. 223229, Sept.2002.

[5] C. Li and S. Roy. Subspace-based blind channel estimation for ofdm by exploiting virtual carriers. IEEE Trans. Wireless Commun., 2(1), Jan. 2003.

[6] R. Steele. Mobile Radio Communications. New York: Wiley, 1974.

[7] S. W. McLanughln Y. Li M. A. Ingram G. L. Stuber, J. R. Barry and T. G. Pratt.Broadband mimo-ofdm wireless communications. Proceedings of the IEEE, 92(2), Feb.2004.

[8] J. J. van de Beek S. K. Wilson O. Edfors, M. Sandell and P. O. Borjesson. Ofdm channel estimation by singular value decomposition. IEEE Trans. Commun., 46(7), July 1998.

[9] M. Sandell S. K. Wilson J. J. van de Beek, O. Edfors and P. O. Borjesson. On channel estimation in OFDM systems. In Proc. IEEE Vehicular Technology Conf., volume 2,pages 815\{819, Chicago, IL., July 1995.

[10] "Mean Squared Error", July 23, 2010. [online] Available: http://en.wikipedia.org/wiki/Mean_squared_error [Accessed: August 2010].

[11] "Symbol Error Rate" for 16-QAM. October 18, 2008. [Online]. Available: http://www.dsplog.com/2007/12/09/symbol-error-rate-for-16 qam/ [Accessed: August 2010]. 